\title{
Multimodal Assessments Are Needed for Restaging after Neoadjunvant Chemoradiation Therapy in Rectal Cancer Patients
}

\author{
Bong-Hyeon Kye, MD, $\mathrm{PhD}^{1}$ \\ Hyung-Jin Kim, MD, PhD ${ }^{1}$ \\ Gun Kim, MD 1 \\ Jun-Gi Kim, MD, PhD2 \\ Hyeon-Min Cho, MD, PhD'
}

${ }^{1}$ Department of Surgery,

St. Vincent's Hospital, College of Medicine,

The Catholic University of Korea, Suwon,

${ }^{2}$ Department of Surgery,

Seoul St. Mary's Hospital,

College of Medicine,

The Catholic University of Korea, Seoul, Korea

\author{
Correspondence: Hyeon-Min Cho, MD, PhD \\ Department of Surgery, St. Vincent's Hospital, \\ College of Medicine, \\ The Catholic University of Korea, \\ 93 Jungbu-daero, Paldal-gu, Suwon 16247, Korea \\ Tel: 82-31-249-7170 \\ Fax: 82-31-247-5347 \\ E-mail: hmcho@catholic.ac.kr \\ Received March 21, 2015 \\ Accepted July 7, 2015 \\ Published Online August 10, 2015
}

\begin{abstract}
Purpose
Restaging after neoadjuvant treatment is done for planning the surgical approach and, increasingly, to determine whether additional therapy or resection can be avoided for selected patients.
\end{abstract}

\begin{abstract}
Materials and Methods
Local restaging after neoadjuvant chemoradiation therapy (nCRT) was performed in 270 patients with locally advanced (cT3or4 or $\mathrm{N}+$ ) rectal cancer. Abdomen and pelvic computed tomography (APCT) was used in all 270 patients, transrectal ultrasound (TRUS) in 121 patients, and rectal magnetic resonance imaging (MRI) in 65 patients. Findings according to imaging modalities were correlated with pathologic stage using Cohen's kappa $(\mathrm{k})$ to test agreement and intra-class correlation coefficient $\alpha$ to test reliability.
\end{abstract}

\section{Results}

Accuracy for prediction of yp T stage according to three imaging modalities was $45.2 \%$ $(\mathrm{K}=0.136, \alpha=0.380)$ in APCT, 49.2\% ( $\mathrm{k}=0.259, \alpha=0.514)$ in rectal MRI, and $57.9 \%$ $(K=0.266, \alpha=0.520)$ in TRUS. Accuracy for prediction of yp $N$ stage was $66.0 \%(K=0.274$, $\alpha=0.441)$ in APCT, $71.8 \%(\kappa=0.401, \alpha=0.549)$ in rectal MRI, and $66.1 \%(\kappa=0.147, \alpha=0.272)$ in TRUS. Of 270 patients, 37 (13.7\%) were diagnosed as pathologic complete responder after nCRT. Rectal MRI for restaging did not predict complete response. On the other hand, TRUS did predict three complete responders $(\kappa=0.238, \alpha=0.401)$.

\section{Conclusion}

APCT, rectal MRI, and TRUS are unreliable in restaging rectal cancer after nCRT. We think that multimodal assessment with rectal MRI and TRUS may be the best option for local restaging of locally advanced rectal cancer after nCRT.

\section{Key words}

Rectal neoplasms, Neoadjuvant therapy, Neoplasm staging

\section{Introduction}

According to some recent reports, neoadjuvant chemoradiation therapy (nCRT) followed by curative surgery has become the therapy of choice in locally advanced rectal cancer. nCRT was introduced to improve local disease control, and several large randomized trials have demonstrated a significant benefit from this treatment, with reduced local recurrence rates and improved survival. Currently, com- bined-modality therapy including surgery, radiotherapy, and chemotherapy is recommended for the majority of patients with stage II or III rectal cancer [1-3].

In the diagnostic work-up of rectal cancer, several imaging techniques are available for staging. Staging is built on two principals. These are defining the local anatomy, allowing for surgical planning, and estimating the prognostic stage by revealing any distant metastases. In most rectal cancer patients, surgical decisions are based on initial stage at diagnosis but may be altered according to response to nCRT [4]. 
Downstaging after nCRT may permit sphincter or even organ-preserving approaches including close follow-up, "wait-and-see." without resection [5-9], while upstaging after nCRT may require more aggressive approaches such as multivisceral resection [10]. However, according to many investigators, imaging modalities including abdomen and pelvic computed tomography (APCT), transrectal ultrasound (TR US), rectal magnetic resonance imaging (MRI), or positron emission tomography computed tomography (PET-CT) for restaging after nCRT have very low accuracy [11-14].

In the current study, we evaluated the accuracy of APCT, rectal MRI, and TRUS for local restaging after nCRT and investigated the best modality for local restaging after nCRT.

\section{Materials and Methods}

Data from patients diagnosed with adenocarcinoma of the rectum less than $12 \mathrm{~cm}$ from the anal verge by rigid proctosigmoidoscopy before nCRT in St. Vincent's Hospital, The Catholic University of Korea, between January 2007 and December 2013 were collected. Among these patients, 270 patients were enrolled in this study. After obtaining review board approval from our institute (VC14RISI0160), the data and clinical information of these 270 patients were analyzed retrospectively.

All 270 patients had clinical T3 to T4 or N positive tumors. All of the analyzed patients underwent neoadjuvant radiotherapy with conventional fractionation, as follows: $1.8 \mathrm{~Gy}$ per day; five fractions per week; and a total dose of 50.4 Gy/28 fractions ( 45 Gy/25 fractions initially to the whole pelvis, followed by $5.4 \mathrm{~Gy} / 3$ fractions as a boost to the gross tumor). All patients received two cycles of concurrent chemotherapy with radiotherapy (5-fluorouracil [5-FU], 400 $\mathrm{mg} / \mathrm{m}^{2}$ intravenously 1 hour before radiotherapy and leucovorin, $20 \mathrm{mg} / \mathrm{m}^{2}$ intravenously immediately before each dose of 5-FU on days 1-5 and days 29-33). Surgical treatment was performed within 6 to 8 weeks after the end of neoadjuvant therapy in all patients.

Imaging studies for restaging after nCRT were performed approximately 4 weeks after completion of radiotherapy. Since nCRT was applied to rectal cancer patients in our institute, all rectal cancer patients who received nCRT have been re-evaluated routinely before radical surgery by contrast-enhanced APCT. We added TRUS in July 2010 and rectal MRI in February 2012 as a routine preoperative procedure for restaging in rectal cancer patients who received nCRT. Consequently, APCT was performed in all 270 patients, TRUS in 121 patients, and MRI in 65 patients. Considering imaging modalities performed in individual patients, only
APCT was performed in 144 patients for restaging, APCT and TRUS in 61 patients, and APCT, TRUS, and rectal MRI in 60 patients, and APCT and rectal MRI were performed for restaging in five patients who were not able to undergo TRUS because of luminal stricture by tumor. All APCT and rectal MRI were reviewed by two radiologists and all TRUS by two colorectal surgeons.

For T staging in TRUS, a clinical complete response of the primary lesion after nCRT was defined when the primary lesion after nCRT was movable and homogenous hypoechoic, remarkably reduced in size, had an intact whole rectal wall structure (especially hyper-echoic submucosal line) which was destructed before nCRT, and did not infiltrate to perirectal tissue (Fig. 1).

For node characterization in APCT and rectal MRI, a combination of short axis dimension greater than $5 \mathrm{~mm}$ and node morphology was used in prediction of nodal involvement. For node characterization in TRUS, hypoechoic lymph nodes with an irregular contour or greater than $5 \mathrm{~mm}$ were defined as metastatic lymph nodes. In the current study, clinical N positive was defined when lymph node involvement was suspected by each imaging modality.

Our data were analyzed using the reference standard of pathologic findings. Radiologic findings were correlated with pathologic stage using Cohen's unweighted kappa (к) to test agreement and intra-class correlation coefficient $\alpha$ to test reliability. $\mathrm{k}$-values of 0.2 to 0.4 indicate fair agreement, 0.4 to 0.6 indicate moderate agreement, and greater than 0.6 indicate excellent agreement. $\mathrm{K} \geq 0.5$ was considered acceptable for the ability to predict pathologic findings. If $\alpha$-value $\geq 0.5$, the result was considered acceptable. Continuous variables were compared using Student's $t$ test and expressed as the mean \pm standard deviation. Categorical variables were analyzed using the $\chi^{2}$ test. Significance was defined as a $p$-value of $\leq 0.05$. All statistical analyses were performed using the SPSS ver. 12.0 for Windows (SPSS Inc., Chicago, IL).

\section{Results}

Of the 270 patients, 168 were males, and 102 were females. The mean age was $62.8 \pm 10.8$ years (range, 34 to 84 years). None of our patients experienced any radiation-related or chemotherapy-related major adverse effects more serious than a grade 3 reaction, according to the Common Toxicity Criteia ver. II.

Table 1 shows the relationship between predicted $\mathrm{T}$ stage (ycT) according to each imaging modality used for restaging after nCRT and pathologic T stage (ypT) after surgery. 

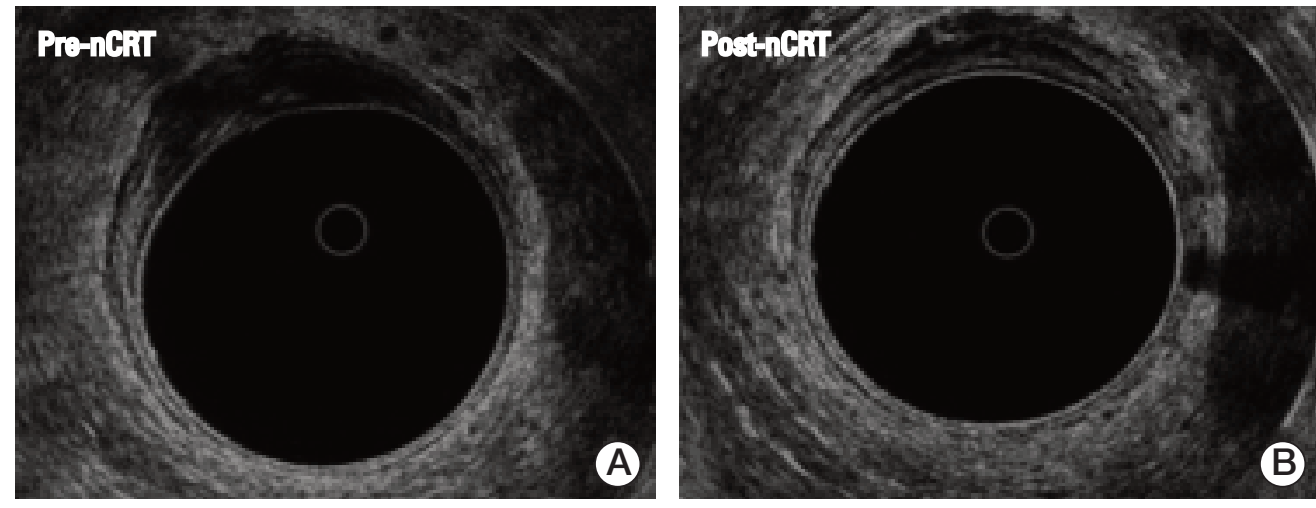

Fig. 1. A clinical complete response of the primary lesion after neoadjuvant chemoradiation therapy (nCRT) was defined when the primary lesion after nCRT was homogenous hypoechoic, reduced in size, had an intact whole rectal wall structure (B), especially hyper-echoic submucosal line, which was destructed before nCRT (A), and did not infiltrate to perirectal tissue.

Table 1. Analysis of the agreement between T stage according to the imaging modalities used for restaging after neoadjuvant therapy and pathologic $\mathrm{T}$ stage after surgery

\begin{tabular}{lcccccc} 
T stage & Under-stage & Agreement & Over-stage & $\mathbf{k}$ & $\boldsymbol{\alpha}$ & $\mathbf{p}$-value \\
APCT & $41(15.2)$ & $122(45.2)$ & $107(39.6)$ & 0.136 & 0.380 & $<0.0001$ \\
Rectal MRI & $7(10.8)$ & $32(49.2)$ & $26(40)$ & 0.259 & 0.514 & 0.002 \\
TRUS & $9(7.4)$ & $70(57.9)$ & $42(34.7)$ & 0.266 & 0.520 & $<0.0001$ \\
\hline
\end{tabular}

Values are presented as number $(\%)$. APCT, abdomen and pelvic computed tomography; MRI, magnetic resonance imaging; TRUS, trans-rectal ultrasound.

Table 2. Analysis of the agreement between $\mathrm{N}$ stage according to the imaging modalities used for restaging and pathologic lymph node involvement after surgery

\begin{tabular}{lcccccc} 
N stage & Under-stage & Agreement & Over-stage & $\mathbf{k}$ & $\boldsymbol{\alpha}$ & $\mathbf{p}$-value \\
APCT & $30(11.1)$ & $178(66.0)$ & $62(23.0)$ & 0.274 & 0.441 & $<0.0001$ \\
Rectal MRI & $8(12.5)$ & $46(71.8)$ & $10(15.6)$ & 0.401 & 0.549 & 0.001 \\
TRUS & $30(24.8)$ & $80(66.1)$ & $11(9.1)$ & 0.147 & 0.272 & $<0.0001$ \\
\hline
\end{tabular}

Values are presented as number (\%). APCT, abdomen and pelvic computed tomography; MRI, magnetic resonance imaging; TRUS, trans-rectal ultrasound.

Accuracy for prediction of ypT stage according to three imaging modalities was $45.2 \%(\kappa=0.136, \alpha=0.380)$ in APCT, $49.2 \%(\mathrm{~K}=0.259, \alpha=0.514)$ in rectal MRI, and $57.9 \%(\mathrm{~K}=0.266$, $\alpha=0.520$ ) in TRUS. Although three imaging tools had low accuracy and low agreement, of those three tools, TRUS was at least the most accurate. The majority of errors were due to over-staging in all three modalities.

Table 2 shows the relationship between $\mathrm{N}$ stage $(\mathrm{ycN})$ according to the imaging modalities used for restaging after
nCRT and pathologic $\mathrm{N}$ stage (ypN) after surgery. Accuracy for prediction of yp $\mathrm{N}$ stage was $66.0 \%(\mathrm{~K}=0.274, \alpha=0.441)$ in APCT, $71.8 \%(\mathrm{~K}=0.401, \alpha=0.549)$ in rectal MRI, and $66.1 \%$ $(\kappa=0.147, \alpha=0.272)$ in TRUS. Errors were mainly caused by over-staging in APCT and rectal MRI and under-staging in TRUS.

Accuracy for overall $\mathrm{T}$ and $\mathrm{N}$ stage is shown in Table 3. Rectal MRI ( $\mathrm{k}=0.326, \alpha=0.613$ ) was the most reliable tool among three modalities for prediction of overall $\mathrm{T}$ and $\mathrm{N}$ 
Table 3. Analysis of the agreement between $\mathrm{T}$ and $\mathrm{N}$ stage according to the imaging modalities used for restaging and pathologic $\mathrm{T}$ and $\mathrm{N}$ stage

\begin{tabular}{lcccccc} 
T and N stage & Under-stage & Agreement & Over-stage & $\mathbf{k}$ & $\boldsymbol{\alpha}$ & $\mathbf{p}$-value \\
APCT & $56(20.7)$ & $89(32.9)$ & $103(38.1)$ & 0.144 & 0.521 & $<0.0001$ \\
Rectal MRI & $11(16.9)$ & $30(46.2)$ & $24(36.9)$ & 0.326 & 0.613 & $<0.0001$ \\
TRUS & $35(28.9)$ & $48(39.6)$ & $38(31.5)$ & 0.203 & 0.315 & 0.020 \\
\hline
\end{tabular}

Values are presented as number $(\%)$. APCT, abdomen and pelvic computed tomography; MRI, magnetic resonance imaging; TRUS, trans-rectal ultrasound.

Table 4. Prediction of complete response

\begin{tabular}{|rrrrrr} 
& yp CR & yp RD & p-value & K & $\alpha$ \\
\hline APCT & & & & & \\
\hline yc CR & 2 & 6 & 0.301 & 0.042 & 0.087 \\
\hline yc RD & 35 & 227 & & & \\
Rectal MRI & & & & & \\
yc CR & 0 & 0 & Not measured & \\
yc RD & 8 & 57 & & & \\
TRUS & & & & & \\
\hline yc CR & 3 & 2 & 0.016 & 0.238 & 0.401 \\
\hline yc RD & 13 & 103 & & & \\
\hline
\end{tabular}

$\mathrm{CR}$, complete response; $\mathrm{RD}$, residual disease; $\mathrm{APCT}$, abdomen and pelvic computed tomography; MRI, magnetic resonance imaging; TRUS, trans-rectal ultrasound. ${ }^{a} K$ and $\alpha$ statistics could not be measured because rectal MRI failed to predict yc CR.

stage.

In the current study, 37 of 270 patients $(13.7 \%)$ were diagnosed as pathologic complete responder after nCRT. Rectal MRI for re-staging did not predict complete response. That is because T0 state was not predicted by rectal MRI. On the other hand, TRUS did predict three complete responders $(\kappa=0.238, \alpha=0.401)$ (Table 4).

\section{Discussion}

Although several methods for precise staging of rectal cancer have been described, TRUS, rectal MRI, and APCT are the most commonly used. Accurate staging of rectal cancer should ideally determine depth of invasion and presence of lymph node metastases and should determine the resectability of locally advanced tumors. After radiation, however, the interpretation of findings becomes more difficult, and the accuracy of all these examinations decreases.

By previous literature, TRUS, APCT, and rectal MRI in rectal cancer patients without nCRT had an acceptable accuracy rate in predicting the pathologic stage. However, they insisted that the accuracy rates of these imaging modalities after nCRT were decreased [14-16]. In the setting of nCRT, studies using APCT have reported accuracy of $46.3 \%$ to $74 \%$ for prediction of ypT stage and 35\% to $76 \%$ for prediction of ypN stage. Studies using TRUS have reported $38.3 \%$ to $92.8 \%$ for prediction of ypT stage and $72.6 \%$ for prediction of ypN stage $[15,16]$. In addition, some investigators demonstrated that standard MRI is not sufficient for restaging after nCRT [14]. These authors explained that the accuracy of radiological restaging after nCRT is impaired due to the difficulty in differentiating fibrotic or reactive tissue from residual tumor.

Lymph node staging with TRUS remains difficult and is less accurate than T staging [17-19]. Staging of bulky, distal and/or stenotic lesion with TRUS can be challenging due to the limited field of view and the inability of probes to transverse the lesion. In addition, TRUS can evaluate only perirectal or mesorectal lymph nodes, thereby limiting the screening capacity of this method. In contrast, other imaging modalities such as APCT and rectal MRI can visualize the iliac and mesenteric or retroperitoneal nodes, allowing for more comprehensive lymph node staging. For lymph node staging, pre-nCRT rectal MRI also has low accuracy when the size criterion of $5 \mathrm{~mm}$ is used. Kim et al. [20] suggested that a lymph node of size $>5 \mathrm{~mm}$ is a significant risk factor for pelvic recurrence in rectal cancer. In addition to size, some imaging findings such as spiculated or indistinct border and mottled heterogeneous appearance could be useful in prediction of regional lymph node involvement in patients with rectal cancer [21]. However, due to the downsizing effect of radiation, it is difficult to adapt the size criterion for the prediction of lymph node involvement after nCRT, and there are no definite criteria for differentiating metastatic lymph node and irradiated lymph node change upon post-nCRT rectal MRI using morphologic criteria.

In the current study, rectal MRI was the most accurate 
imaging modality for local restaging after nCRT. In particular, rectal MRI can be helpful in determining the circumferential resection margin of total mesorectal excision specimens by providing information on the distance between the outermost edge of the tumor and the mesorectal fascia [22]. However, rectal MRI did fail to predict complete responder. On the other hand, although TRUS was a less accurate imaging modality with a limitation in prediction of ypN stage, it was more accurate than rectal MRI for prediction of pathologic complete responder as well as ypT stage. Statistical values of TRUS for pathologic complete response; the overall accuracy was 106/121 (87.6\%), 18.8\% of sensitivity, $98.1 \%$ of specificity, $60 \%$ of positive predictive value, and $88.8 \%$ of negative predictive value. In our study, five patients were predicted as ycT0 stage by TRUS. All five of these patients were predicted complete responders by TRUS. On pathologic examination, three of these five patients were pathologic complete responders and one was ypT0N1 and the other one was ypT1N1. One of these five patients was predicted ycN+ by MRI. However, pathologic finding indicated that he was a complete responder. In view of a tailored surgical option including local excision for good responder after nCRT, a false-negative diagnosis reflected by a negative predictive value may be crucial for planning a surgical treatment [16].

The limitations of our study are that three imaging modalities were not performed in all patients and that APCT and rectal MRI were interpreted by several radiologists whereas TRUS by a single surgeon. In addition, we did not evaluate the accuracy of PET-CT, which, as recently mentioned, has the potential for superiority in restaging for locally advanced rectal cancer. However, due to limitations of the Korean health care insurance system, routine performance of PETCT for restaging is not practical. We think that a welldesigned large scale study including various imaging studies performed by co-work between radiologist and surgeon specialized in colorectal cancer is needed.

\section{Conclusion}

Given the low correspondence between pathologic status prediction and actual pathologic assessment, each imaging modality including CT, MRI, and TRUS is unreliable in restaging rectal cancer after nCRT. Restaging after nCRT is challenging with all modalities due to difficulty in reliably differentiating between radiation-induced changes and the tumor itself. However, based on our results, rectal MRI might be helpful for overall tumor condition after nCRT and TRUS for predicting complete responder. We think that rectal MRI and TRUS may be complementary, not alternative options for local restaging of locally advanced rectal cancer after nCRT.

\section{Conflicts of Interest}

Conflict of interest relevant to this article was not reported.

\section{References}

1. Sauer R, Becker H, Hohenberger W, Rodel C, Wittekind C, Fietkau R, et al. Preoperative versus postoperative chemoradiotherapy for rectal cancer. N Engl J Med. 2004;351:1731-40.

2. Kapiteijn E, Marijnen CA, Nagtegaal ID, Putter H, Steup WH, Wiggers T, et al. Preoperative radiotherapy combined with total mesorectal excision for resectable rectal cancer. N Engl J Med. 2001;345:638-46.

3. Theodoropoulos G, Wise WE, Padmanabhan A, Kerner BA, Taylor CW, Aguilar PS, et al. T-level downstaging and complete pathologic response after preoperative chemoradiation for advanced rectal cancer result in decreased recurrence and improved disease-free survival. Dis Colon Rectum. 2002;45: 895-903.

4. Van Cutsem E, Dicato M, Haustermans K, Arber N, Bosset JF, Cunningham $\mathrm{D}$, et al. The diagnosis and management of rectal cancer: expert discussion and recommendations derived from the 9th World Congress on Gastrointestinal Cancer, Barcelona, 2007. Ann Oncol. 2008;19 Suppl 6:vi1-8.

5. Kim CJ, Yeatman TJ, Coppola D, Trotti A, Williams B, Barthel JS, et al. Local excision of T2 and T3 rectal cancers after downstaging chemoradiation. Ann Surg. 2001;234:352-8.

6. Kennelly RP, Heeney A, White A, Fennelly D, Sheahan K, Hyland JM, et al. A prospective analysis of patient outcome following treatment of T3 rectal cancer with neo-adjuvant chemoradiotherapy and transanal excision. Int J Colorectal Dis. 2012;27:759-64.

7. Habr-Gama A, Perez RO, Nadalin W, Sabbaga J, Ribeiro U Jr, Silva e Sousa AH Jr, et al. Operative versus nonoperative treatment for stage 0 distal rectal cancer following chemoradiation therapy: long-term results. Ann Surg. 2004;240:711-7.

8. Martin ST, Heneghan HM, Winter DC. Systematic review and meta-analysis of outcomes following pathological complete 
response to neoadjuvant chemoradiotherapy for rectal cancer. Br J Surg. 2012;99:918-28.

9. Smith FM, Waldron D, Winter DC. Rectum-conserving surgery in the era of chemoradiotherapy. Br J Surg. 2010;97: 1752-64.

10. Gannon CJ, Zager JS, Chang GJ, Feig BW, Wood CG, Skibber JM, et al. Pelvic exenteration affords safe and durable treatment for locally advanced rectal carcinoma. Ann Surg Oncol. 2007;14:1870-7.

11. Dresen RC, Beets GL, Rutten HJ, Engelen SM, Lahaye MJ, Vliegen RF, et al. Locally advanced rectal cancer: MR imaging for restaging after neoadjuvant radiation therapy with concomitant chemotherapy. Part I. Are we able to predict tumor confined to the rectal wall? Radiology. 2009;252:71-80.

12. Barbaro B, Vitale R, Leccisotti L, Vecchio FM, Santoro L, Valentini $\mathrm{V}$, et al. Restaging locally advanced rectal cancer with MR imaging after chemoradiation therapy. Radiographics. 2010; 30:699-716.

13. Pomerri F, Pucciarelli S, Maretto I, Zandona M, Del Bianco P, Amadio L, et al. Prospective assessment of imaging after preoperative chemoradiotherapy for rectal cancer. Surgery. 2011;149:56-64.

14. Hanly AM, Ryan EM, Rogers AC, McNamara DA, Madoff RD, Winter DC, et al. Multicenter Evaluation of Rectal cancer ReImaging pOst Neoadjuvant (MERRION) therapy. Ann Surg. 2014;259:723-7.

15. Barbaro B, Schulsinger A, Valentini V, Marano P, Rotman M. The accuracy of transrectal ultrasound in predicting the pathological stage of low-lying rectal cancer after preoperative chemoradiation therapy. Int J Radiat Oncol Biol Phys. 1999; 43:1043-7.

16. Huh JW, Park YA, Jung EJ, Lee KY, Sohn SK. Accuracy of endorectal ultrasonography and computed tomography for restaging rectal cancer after preoperative chemoradiation. J Am Coll Surg. 2008;207:7-12.

17. Herzog U, von Flue M, Tondelli P, Schuppisser JP. How accurate is endorectal ultrasound in the preoperative staging of rectal cancer? Dis Colon Rectum. 1993;36:127-34.

18. Garcia-Aguilar J, Pollack J, Lee SH, Hernandez de Anda E, Mellgren A, Wong WD, et al. Accuracy of endorectal ultrasonography in preoperative staging of rectal tumors. Dis Colon Rectum. 2002;45:10-5.

19. Lin S, Luo G, Gao X, Shan H, Li Y, Zhang R, et al. Application of endoscopic sonography in preoperative staging of rectal cancer: six-year experience. J Ultrasound Med. 2011;30: 1051-7.

20. Kim TH, Jeong SY, Choi DH, Kim DY, Jung KH, Moon SH, et al. Lateral lymph node metastasis is a major cause of locoregional recurrence in rectal cancer treated with preoperative chemoradiotherapy and curative resection. Ann Surg Oncol. 2008;15:729-37.

21. Kim JH, Beets GL, Kim MJ, Kessels AG, Beets-Tan RG. Highresolution MR imaging for nodal staging in rectal cancer: are there any criteria in addition to the size? Eur J Radiol. 2004; 52:78-83.

22. MERCURY Study Group. Extramural depth of tumor invasion at thin-section MR in patients with rectal cancer: results of the MERCURY study. Radiology. 2007;243:132-9. 